\title{
USING OPTICAL ENHANCEMENTS OF INFRARED MICROSPECTROSCOPY TO ADVANTAGE
}

\author{
David L. Wetzel \\ Microbeam Molecular Spectroscopy Laboratory, Kansas State University, \\ Shellenberger Hall, Manhattan, KS 66506-2201, USA
}

In the past a number of commercial mirror lens objectives have been made available that have enhanced the acquisition of data with infrared microspectroscopy. This includes special objectives for internal reflection (ATR) to allow surface preferenced analysis. A grazing angle objective that is useful for analyzing thin films that are on a reflective surface. An infrared microscope operating in the reflection mode can use a technique of absorption reflection absorption as well as diffuse reflectance. Compression cells are used to flatten specimens. This is particularly useful for mapping experiments to provide uniform focus at all parts of the specimen.

Commonly used non hygroscopic dispersive substrates for infrared microspectroscopy are dispersive resulting in a different infrared focus, at various wavelengths. The infrared focus is in fact not coincident with the visible focus. The problem is particularly troublesome at the longer wavelengths. This has been discussed in the past by Carr (1). A simple optical enhancement is to use a man-made diamond window, which is available $1 \mathrm{~mm}$ thick and $4 \mathrm{~mm}$ in diameter, for less than the cost of 10 barium fluoride disks. Although, the diamond does not compete optically with silver chloride, which has been available for a number of years, the silver chloride is seldom used, since it is very soft and photosensitive.

One enhancement of the signal to noise ratio that is useful for analyzing relatively small quantities of a species with fairly weak absorptions has proved useful for analyzing deuterated compounds. In the biological field, a useful way of doing metabolism studies involves the deuterium mass isotope rather than a radioactive isotope. In the material sciences, it is convenient to use deuterated compounds for polymer permeation studies. Synchrotron infrared microspectroscopy represents a three-fold source enhancement that is particularly useful for microspectroscopy. The increased brightness in comparison to a thermal source and the absence of thermal noise with synchrotron radiation, results in an excellent signal to noise ratio. In infrared microspectroscopy, of a remote projected image plane mask usually results in a sizeable loss of signal. However, because the synchrotron radiation is non-dispersive as much as $85 \%$ of the radiation encountered without an image plane mask made its way through an 8.5 micrometer pinhole.

Polarization infrared is useful to determine molecular orientation based on the observation of dichroism. Where the absorption band of a particular functional group that is oriented has a different response when the polarizer is parallel to the axis of the chain (such as in a single fiber), in comparison to the response when it is perpendicular. A useful enhancement to extensive polarization studies is the use of a rotating microscope stage. This enables the operator to use a single background at each polarizer orientation, while studying the dichroic effects of rotation of the sample to different angles from that of the axial radiation through a highly molecularly oriented specimen. 
Locating the area of interest in the microscopic field of view is sometimes difficult in the absence of the use of stain. Verification of targets in the infrared is often done by examining stained adjacent contiguous sections. This practice makes the spectroscopic response more acceptable to microscopists in the biological field who are familiar with histology. In the absence of the stain it is possible to produce differential interference contrast. This involves the use of a polarizer and a pair of wollesten prisms before the stage followed by a pair of prisms and an analyzer after the stage. This enhancement is made possible when infinity corrected optics are employed. The differential interference contrast is produced from the illuminating white light. Once the locations of different features in the field have been established, it is necessary to remove the prisms and polarizers prior to performing the spectroscopic analysis.

In some instances fluorescence assisted infrared microspectroscopy has been employed. In particular at beamline U10B, at the National Synchrotron Light Source, (NSLS) of Brookhaven National Laboratory (BNL) at Upton, NY. This optical enhancement has been used in connection with fluorophor reagents by mounting the specimen in a single instrument that is equipped for photographing the fluorescence observations before subsequent infrared microspectroscopic analysis. This practice removes any doubt as to the location of the tissue to be targeted and first proved useful in bone studies.

Examples of the use of a number of these optical enhancements are presented. It is obvious that infrared microspectroscopists have a number of tools in the toolbox that make it possible to extend the modern discipline to a large number of diverse analytical problems.

\section{Acknowledgement}

Space does not allow references to each enhancement. We thank the innovators from commercial instrument companies, and those from the ranks of instrument scientists and practicing infrared microspectroscopists for whom necessity was the mother of invention. We all may benefit from these enhancements.

Contribution No. 03-PXXX Kansas Agricultural Experimental Station, Manhattan.

References

Carr, G.L., Rev. Sci. Instr., 2001, 72(3):1613-1619. 